PROCEEDINGS OF THE

AMERICAN MATHEMATICAL SOCIETY

Volume 131, Number 10, Pages 3191-3201

S 0002-9939(03)07055-2

Article electronically published on April 30, 2003

\title{
THE SELF-SIMILAR EXPANDING CURVE FOR THE CURVATURE FLOW EQUATION
}

\author{
HUA-HUAI CHERN, JONG-SHENQ GUO, AND CHU-PIN LO
}

(Communicated by David S. Tartakoff)

\begin{abstract}
We study a two-point free boundary problem for the curvature flow equation. By studying the corresponding nonlinear initial value problem, we obtain the existence and uniqueness of the forward self-similar solution of this problem. The corresponding curve is called the self-similar expanding curve. We also derive the asymptotic stability of this curve.
\end{abstract}

\section{INTRODUCTION}

Moving phase boundary (interface) is a very interesting phenomenon observed in material science, crystalline growth, chemical reaction, physiology system, and so on. For example, in crystalline growth phenomenon, interfaces are boundaries separating two different phases, liquid and solid. In an excitable physiology system, such as the heart system, there occur many interesting wave patterns, such as traveling fronts, spiral waves, and scroll waves, which also represent the interfaces separating different phases, rest, excitation, and refractory states. There have been many approaches proposed to study the motions of interfaces. One famous model is the equation of the following form:

$$
v=F(\kappa, \nu),
$$

where $v$ is the normal velocity of the interface, $\kappa$ is the curvature, and $\nu$ is the outward normal vector of the interface. We refer the readers to $[13$ for the derivation of this model. In particular, when $F(\kappa, \nu)=\kappa$, this is the classical curvature flow (23]); when $F(\kappa, \nu)=c-D \kappa$, for some constants $c$ and $D$, this is the so-called eikonal curvature relation in the excitable physiology system ([17]), or the governing equation of crystal growth ([4]). For other applications of (1.1), we refer the readers to [11, 16, 19, 23.

To study (1.1), many methods have been proposed. In the level set method (see [6, 9, 23]), one assumes that an interface is a level set of some function $u$ which usually represents the concentration of some chemical element in a chemical reaction system. Using (1.1), some partial differential equations of $u$ can be derived. Then the behavior of the interface can be studied. In other methods, one seeks for a parametrization [19, graph representation [1, 2, 15, 18, 20, 21], or auxiliary function 7] of the interface and then studies some alternative equations coming from (1.1).

Received by the editors May 16, 2002.

2000 Mathematics Subject Classification. Primary 35B60, 34A12, 35B35.

(C)2003 American Mathematical Society 
In [15, Huisken proved that graphs $\{(x, u(x, t)) \mid x \in \Omega\}, \Omega$ a bounded domain in $R^{2}$, evolving by a non-parametric curvature flow with vertical contact angle to a fixed boundary, converge to constant functions. In [1], Altschuler and Wu proved that one-dimensional graphs $\{(x, u(x, t)) \mid x \in[0, d]\}(d<\infty)$ evolving by a general class of quasilinear equations with prescribed contact angle to a fixed boundary converge to translating solitons. Later in [2], they considered two-dimensional graphs $\{(x, u(x, t)) \mid x \in \Omega\}, \Omega$ a bounded domain in $R^{2}$, governed by the mean curvature flow equation with prescribed contact angle to a fixed boundary. They proved that such graphs also converge to some surface which moves at a constant speed. In 18, Kohsaka considered one-dimensional graphs evolving by a general class of quasilinear equations on a domain with one free end. In 20 21, Ninomiya and Taniguchi considered one-dimensional graph representations of interfaces on the whole real line for $F(\kappa, \nu)=\kappa+c$, where $c$ is a nonzero constant. They focused on the so-called traveling curved fronts, such as the $\mathrm{V}$-shaped waves which have been observed in many experiments and obtained various existence and asymptotic convergence results. See also 8 .

In this paper, we take $F(\kappa, \nu)=\kappa$, use one-dimensional graph representation to locate the position of the interface, and consider the problem with prescribed touching angles to two free ends. We study the following two-point free boundary problem for the curvature flow equation:

$$
\left\{\begin{array}{l}
u_{t}=u_{x x} /\left(1+u_{x}^{2}\right), \quad-\xi_{1}(t)<x<\xi_{2}(t), t>0 \\
u_{x}\left(-\xi_{1}(t), t\right)=\tan \left(\theta_{1}-\beta_{1}\right), \quad u\left(-\xi_{1}(t), t\right)=\xi_{1}(t) \tan \beta_{1}, t>0 \\
u_{x}\left(\xi_{2}(t), t\right)=\tan \left(\beta_{2}-\theta_{2}\right), \quad u\left(\xi_{2}(t), t\right)=\xi_{2}(t) \tan \beta_{2}, t>0 \\
u(x, 0)=u_{0}(x),-\xi_{1}(0) \leq x \leq \xi_{2}(0), \quad \xi_{1}(0)=\xi_{01}, \quad \xi_{2}(0)=\xi_{02}
\end{array}\right.
$$

where $\beta_{i}$ and $\theta_{i}$ are given constants satisfying $\beta_{i} \in[0, \pi / 2)$ and $\theta_{i} \in\left(0, \beta_{i}+\right.$ $\pi / 2), i=1,2, \xi_{01}$ and $\xi_{02}$ are positive constants, $u_{0} \in C^{2}\left[-\xi_{01}, \xi_{02}\right]$ satisfying the compatibility conditions, and $u_{0}>0$ in $\left(-\xi_{01}, \xi_{02}\right)$. In this problem, $u, \xi_{1}, \xi_{2}$ are unknown functions to be determined.

For a solution $\left(u, \xi_{1}, \xi_{2}\right)$ of (1.2), we define the corresponding curve by

$$
\Gamma(t):=\left\{(x, u(x, t)) \mid-\xi_{1}(t) \leq x \leq \xi_{2}(t)\right\} .
$$

Set $\partial \Omega_{1}:=\left\{(x, z) \mid z=-\left(\tan \beta_{1}\right) x, x \leq 0\right\}$ and $\partial \Omega_{2}:=\left\{(x, z) \mid z=\left(\tan \beta_{2}\right) x, x \geq\right.$ $0\}$. Let $D(t)$ be the domain enclosed by $\Gamma(t), \partial \Omega_{1}$, and $\partial \Omega_{2}$. By a simple calculation and using the relation $u_{x x} /\left(1+u_{x}^{2}\right)=\left[\tan ^{-1}\left(u_{x}\right)\right]_{x}$, we have

$$
\frac{d}{d t} \mu(D(t))=\alpha_{2}-\alpha_{1}
$$

where $\alpha_{1}:=\theta_{1}-\beta_{1}, \alpha_{2}:=\beta_{2}-\theta_{2}$, and $\mu(D)$ is the area of $D$. This implies that

$$
\frac{d}{d t} \mu(D(t))\left\{\begin{array}{lll}
>0 & \text { if } & \alpha_{1}<\alpha_{2} \\
=0 & \text { if } & \alpha_{1}=\alpha_{2} \\
<0 & \text { if } & \alpha_{1}>\alpha_{2}
\end{array}\right.
$$

We are interested in the case when $\alpha_{1}<\alpha_{2}$. This is the so-called expanding case. We are looking for the forward self-similar solution of (1.2) in the form

$$
U(z, t):=\sqrt{2(t+1)} \varphi\left(\frac{z}{\sqrt{2(t+1)}}\right)
$$


for some smooth function $\varphi$. Then $U$ satisfies (1.2) if and only if $\varphi$ satisfies the following nonlinear free boundary problem:

$$
\begin{aligned}
& \frac{\varphi^{\prime \prime}(y)}{1+\left(\varphi^{\prime}(y)^{2}\right.}+y \varphi^{\prime}(y)-\varphi(y)=0, \quad-p<y<q, \\
& \varphi(-p)=p \tan \beta_{1}, \quad \varphi^{\prime}(-p)=\tan \alpha_{1}, \\
& \varphi(q)=q \tan \beta_{2}, \quad \varphi^{\prime}(q)=\tan \alpha_{2}
\end{aligned}
$$

for some positive constants $p$ and $q$. We call the corresponding curve

$$
S(t):=\{(z, U(z, t)) \mid-\sqrt{2(t+1)} p \leq z \leq \sqrt{2(t+1)} q\}
$$

a self-similar expanding curve. To study the existence of a self-similar expanding curve, we study the initial value problem for (1.5)-(1.6) (denoted by (IVP)) for any given $p>0$.

In [12, the authors studied a problem similar to (1.2) in which the nonlinear operator is replaced by the heat operator. The problem arises in the combustion theory and is motivated by the mathematical modeling of flame propagation (cf. [3]). In [12], the existence, uniqueness, and asymptotic stability of the self-similar expanding curve is investigated. Since the equation is linear, the solution of the corresponding initial value problem can be expressed explicitly. Hence the existence and uniqueness of self-similar expanding curve can be readily derived.

In our case, since the equation is nonlinear, we need to study the existence interval more carefully. Indeed, the solution of (IVP) cannot be extended beyond $y=0$ if $p$ is sufficiently large. Moreover, there are small $p>0$ such that the solution of (IVP) can be defined for all $y>-p$. It is interesting to note that the (local) solution of (IVP) is global as long as the (local) solution is defined beyond $y=0$. By applying a comparison principle for nonlinear equations, we are able to derive the existence and uniqueness of the self-similar expanding curve. Furthermore, the stability of this self-similar expanding curve can be proved by the same argument as the one given by Kohsaka in [18. (see also [12]).

We remark that the existence of so-called self-similar shrinking curves for the heat equation is also studied in [12. In 18, the one-point free boundary problem for the quasilinear parabolic equation

$$
u_{t}=\left(a\left(u_{x}\right)\right)_{x}
$$

where $a \in C^{2}(\mathbf{R}), a^{\prime}(s)>0$ for $s \in \mathbf{R}$, was studied (see also the references cited therein). Here the boundary conditions are given by

$$
u_{x}(s(t), t)=\tan \theta_{1}, u(s(t), t)=0, u_{x}(0, t)=\tan \theta_{2}
$$

with $\theta_{i} \in(0, \pi / 2), i=1,2$. In [18], the author obtained the existence, uniqueness, and asymptotic stability of the self-similar expanding curve when $\theta_{1}<\theta_{2}$. For other related problems, we refer the readers to the papers of Caffarelli-Vazquez [5], Galaktionov-Hulshof-Vazquez [10], Hilhorst-Hulshof [14], and Vazquez [24].

This paper is organized as follows. We first prove the existence and uniqueness of a self-similar expanding curve of the two-point free boundary problem (1.2) in $\S 2$. Then we study its asymptotic stability in $\S 3$. 


\section{Existence AND Uniqueness}

In this section, we study the existence of the solution $(\varphi, p, q)$ for the following nonlinear free boundary problem $(\mathrm{P})$ :

$$
\begin{aligned}
& \frac{\varphi^{\prime \prime}(y)}{1+\left(\varphi^{\prime}(y)\right)^{2}}+y \varphi^{\prime}(y)-\varphi(y)=0, \quad-p<y<q, \\
& \varphi(-p)=p \tan \beta_{1}, \quad \varphi^{\prime}(-p)=\tan \alpha_{1}, \\
& \varphi(q)=q \tan \beta_{2}, \quad \varphi^{\prime}(q)=\tan \alpha_{2},
\end{aligned}
$$

where $p, q>0, \beta_{1}, \beta_{2} \in[0, \pi / 2), \alpha_{1} \in\left(-\beta_{1}, \pi / 2\right)$, and $\alpha_{2} \in\left(\alpha_{1}, \beta_{2}\right)$.

We introduce the following differential operators:

$$
N[u](y):=u^{\prime \prime}(y)+\mathcal{U}\left(y, u, u^{\prime}\right), \quad \mathcal{U}\left(y, u, u^{\prime}\right):=\left[y u^{\prime}(y)-u(y)\right]\left[1+\left(u^{\prime}(y)\right)^{2}\right] .
$$

Notice that the function $\mathcal{U}(y, u, v)$ satisfies

$$
\frac{\partial \mathcal{U}}{\partial u}=-\left(1+v^{2}\right)<0
$$

We now consider the nonlinear initial value problem:

$$
N[\varphi](y)=0, \quad \varphi^{\prime}(-p)=\tan \alpha_{1}, \quad \varphi(-p)=p \tan \beta_{1} .
$$

Let $\varphi(y ; p)$ be the (local) solution of the nonlinear initial value problem (2.4), where $p>0, \beta_{1} \in[0, \pi / 2)$ and $\alpha_{1} \in\left(-\beta_{1}, \pi / 2\right)$ are given. Define

$$
\mathcal{F}(y):=-y \varphi^{\prime}(y ; p)+\varphi(y ; p), \quad y \geq-p .
$$

Since $\mathcal{F}(-p)=p A_{1}>0, A_{1}:=\tan \beta_{1}+\tan \alpha_{1}$, and

$$
\mathcal{F}^{\prime}(y)=-y \varphi^{\prime \prime}(y ; p)=(-y)\left(1+\left(\varphi^{\prime}(y ; p)\right)^{2}\right) \mathcal{F}(y), \quad y \geq-p,
$$

we have

$$
\mathcal{F}(y)=p A_{1} \exp \left\{\frac{1}{2}\left(p^{2}-y^{2}\right)-\int_{-p}^{y} t\left(\varphi^{\prime}(t ; p)\right)^{2} d t\right\}>0, \quad y \geq-p
$$

From (2.6) and the equality

$$
\frac{\varphi^{\prime \prime}(y ; p)}{1+\left(\varphi^{\prime}(y ; p)\right)^{2}}=\mathcal{F}(y)
$$

it follows that $\varphi^{\prime \prime}(y ; p)>0$ for $y \geq-p$ for any $p>0$.

From now on, we shall use $[-p, R(p))$ to denote the maximal interval of existence of the solution $\varphi(y ; p)$, where $-p<R(p) \leq \infty$. We first study the function $R(p)$ for all $p>0$.

Lemma 2.1. If $p>0$ satisfying $\alpha_{1}+p^{2} A_{1} \geq \pi / 2$, then $R(p) \leq 0$.

Proof. For $y<0$, since $\mathcal{F}^{\prime}(y)>0$ by (2.5), we have $\mathcal{F}(y) \geq p A_{1}$. Then by (2.7)

$$
\tan ^{-1} \varphi^{\prime}(y ; p) \geq \alpha_{1}+p A_{1}(y+p) \text {. }
$$

If $R(p)>0$, then $\varphi^{\prime}(0 ; p)$ is well defined and $\tan ^{-1} \varphi^{\prime}(0 ; p) \geq \pi / 2$ by assumption. This is clearly a contradiction, since the range of $\tan ^{-1}$ is $(-\pi / 2, \pi / 2)$. Hence the lemma is proved.

Lemma 2.2. $R(p)>0$ for some $p>0$. 
Proof. First we assume that $\alpha_{1} \geq 0$. Choose $p>0$ such that

$$
I(p):=\int_{\tan \alpha_{1}}^{\infty} \frac{d \sigma}{\left(1+\sigma^{2}\right) \exp \left\{\frac{1}{2} p^{2}\left(1+\sigma^{2}\right)\right\}}>p^{2} A_{1} .
$$

Note that such $p$ exists, since $I(p)$ is decreasing as $p$ increases and

$$
I(p) \uparrow \int_{\tan \alpha_{1}}^{\infty} \frac{d \sigma}{1+\sigma^{2}}>0 \text { as } p \downarrow 0^{+} .
$$

Let $\varphi(y)$ be the solution of (2.4) with $\alpha_{1} \geq 0$. Recall that $\varphi^{\prime \prime}>0$. Then

$$
\varphi^{\prime}\left(y_{1}\right)^{2}>\varphi^{\prime}\left(y_{2}\right)^{2} \text { if } y_{1}>y_{2} \geq-p .
$$

Suppose that $R(p) \leq 0$. Then, by (2.6), (2.7), and (2.9), for $y \in(-p, R(p))$

$$
\begin{aligned}
\varphi^{\prime \prime}(y) & =\left[1+\varphi^{\prime}(y)^{2}\right] p A_{1} \exp \left\{\frac{1}{2}\left(p^{2}-y^{2}\right)+\int_{-p}^{y}(-t) \varphi^{\prime}(t)^{2} d t\right\} \\
& \leq\left[1+\varphi^{\prime}(y)^{2}\right] p A_{1} \exp \left\{\frac{1}{2} p^{2}\left[1+\varphi^{\prime}(y)^{2}\right]\right\}
\end{aligned}
$$

and so

$$
\frac{\varphi^{\prime \prime}(y)}{\left[1+\varphi^{\prime}(y)^{2}\right] \exp \left\{\frac{1}{2} p^{2}\left[1+\varphi^{\prime}(y)^{2}\right]\right\}} \leq p A_{1}
$$

for all $y \in(-p, R(p))$. Then by integrating (2.10) from $-p$ to $R(p)$ we obtain that

$$
\int_{-p}^{R(p)} \frac{\varphi^{\prime \prime}(y) d y}{\left[1+\varphi^{\prime}(y)^{2}\right] \exp \left\{\frac{1}{2} p^{2}\left[1+\varphi^{\prime}(y)^{2}\right]\right\}} \leq p^{2} A_{1} .
$$

Setting $\sigma:=\varphi^{\prime}(y)$, we deduce that

$$
\int_{\tan \alpha_{1}}^{\infty} \frac{d \sigma}{\left(1+\sigma^{2}\right) \exp \left\{\frac{1}{2} p^{2}\left(1+\sigma^{2}\right)\right\}} \leq p^{2} A_{1},
$$

contradicting (2.8). This implies that $R(p)>0$.

Now, we choose $p>0$ so that $R_{0}(p)>0$, where $\left[-p, R_{0}(p)\right)$ denotes the maximal interval of existence of the solution $\varphi_{0}$ of (2.4) with $\alpha_{1}=0$. Let $\varphi$ be the corresponding solution of (2.4) with $\alpha_{1}<0$. Since $\varphi^{\prime}(-p)<\varphi_{0}^{\prime}(-p)$ and $\varphi(-p)=\varphi_{0}(-p)$, it follows from the standard nonlinear comparison theorem [22] Theorem 23 of Chap. 1] that $\varphi(y) \leq \varphi_{0}(y)$ and $\varphi^{\prime}(y) \leq \varphi_{0}^{\prime}(y)$ for $y \in[-p, R)$, where $R:=\min \left\{R(p), R_{0}(p)\right\}$. Hence $R(p) \geq R_{0}(p)>0$. This proves the lemma.

For each $p>0$ with $R(p)>0$ and $q \in(0, R(p))$, we define

$$
\mathcal{G}(q, p)=\varphi^{\prime}(q ; p), \quad \mathcal{H}(q, p)=\frac{1}{q} \varphi(q ; p) .
$$

We shall discuss the monotonicity of these two functions $\mathcal{G}$ and $\mathcal{H}$. Note that $\mathcal{G}$ is monotone increasing in $q$ and $\mathcal{H}$ is monotone decreasing in $q$, since

$$
\frac{\partial \mathcal{G}}{\partial q}(q, p)=\varphi^{\prime \prime}(q ; p)>0, \quad \frac{\partial \mathcal{H}}{\partial q}(q, p)=-\frac{\varphi^{\prime \prime}(q ; p)}{q^{2}\left[1+\varphi^{\prime}(q ; p)^{2}\right]}<0 .
$$

Proposition 2.3. If $p_{1}>p_{2}>0$ and $R\left(p_{1}\right)>-p_{2}$, then

$$
\varphi\left(y ; p_{1}\right)>\varphi\left(y ; p_{2}\right), \quad \varphi^{\prime}\left(y ; p_{1}\right)>\varphi^{\prime}\left(y ; p_{2}\right)
$$

for all $y \in\left[-p_{2}, R\right)$, where $R=\min \left\{R\left(p_{1}\right), R\left(p_{2}\right)\right\}$. 
Proof. Since $\varphi^{\prime \prime}>0, \varphi^{\prime}\left(y ; p_{1}\right)>\tan \alpha_{1}$ for $y>-p_{1}$. Then by the fundamental theorem of calculus we have

$$
\varphi\left(y ; p_{1}\right)=p_{1} \tan \beta_{1}+\int_{-p_{1}}^{y} \varphi^{\prime}\left(t ; p_{1}\right) d t>p_{1} \tan \beta_{1}+\left(y+p_{1}\right) \tan \alpha_{1}
$$

for $y>-p_{1}$. Hence we have $\varphi^{\prime}\left(-p_{2} ; p_{1}\right)>\varphi^{\prime}\left(-p_{2} ; p_{2}\right)$ and $\varphi\left(-p_{2} ; p_{1}\right)>\varphi\left(-p_{2} ; p_{2}\right)$. Then it follows from the standard nonlinear comparison theorem [22, Theorem 23 of Chap. 1] that $\varphi\left(y ; p_{1}\right) \geq \varphi\left(y ; p_{2}\right)$ and $\varphi^{\prime}\left(y ; p_{1}\right) \geq \varphi^{\prime}\left(y ; p_{2}\right)$ for $y \in\left[-p_{2}, R\right)$.

We now prove that (2.12) holds for all $y \in\left[-p_{2}, R\right)$. By continuity, (2.12) holds for $y \in\left[-p_{2},-p_{2}+\delta\right]$ for some $\delta>0$. If (2.12) does not hold for all $y \in$ $\left[-p_{2}, R\right)$, then there is the smallest $y_{0}>-p_{2}$ such that $\varphi^{\prime}\left(y_{0} ; p_{1}\right)=\varphi^{\prime}\left(y_{0} ; p_{2}\right)$ and $\varphi\left(y_{0} ; p_{1}\right)>\varphi\left(y_{0} ; p_{2}\right)$. Note that $\varphi^{\prime}\left(y ; p_{1}\right)-\varphi^{\prime}\left(y ; p_{2}\right)>0$ for $y \in\left[-p_{2}, y_{0}\right)$ and $\varphi^{\prime}\left(y_{0} ; p_{1}\right)-\varphi^{\prime}\left(y_{0} ; p_{2}\right)=0$. Then $\varphi^{\prime \prime}\left(y_{0} ; p_{1}\right) \leq \varphi^{\prime \prime}\left(y_{0} ; p_{2}\right)$ and so

$$
\begin{aligned}
0 & =\frac{\varphi^{\prime \prime}\left(y_{0} ; p_{1}\right)}{1+\left(\varphi^{\prime}\left(y_{0} ; p_{1}\right)\right)^{2}}+y_{0} \varphi^{\prime}\left(y_{0} ; p_{1}\right)-\varphi\left(y_{0} ; p_{1}\right) \\
& <\frac{\varphi^{\prime \prime}\left(y_{0} ; p_{2}\right)}{1+\left(\varphi^{\prime}\left(y_{0} ; p_{2}\right)\right)^{2}}+y_{0} \varphi^{\prime}\left(y_{0} ; p_{2}\right)-\varphi\left(y_{0} ; p_{2}\right) \\
& =0,
\end{aligned}
$$

a contradiction. This proves the proposition.

As a consequence of Proposition 2.3. we have

$$
R\left(p_{2}\right) \geq R\left(p_{1}\right), \text { if } p_{1}>p_{2}>0 .
$$

Furthermore,

$$
\mathcal{G}\left(q, p_{1}\right)>\mathcal{G}\left(q, p_{2}\right), \mathcal{H}\left(q, p_{1}\right)>\mathcal{H}\left(q, p_{2}\right) \text {, if } p_{0}>p_{1}>p_{2}>0,
$$

where $p_{0}:=\inf \{p>0 \mid R(p) \leq 0\}$. Note that $p_{0}>0$ by Lemma 2.2 and (2.14). Also, $R(p)>0$ for all $p \in\left(0, p_{0}\right)$.

We claim that

$$
R\left(p_{0}\right) \leq 0 .
$$

Otherwise, suppose that $R\left(p_{0}\right)>0$. Consider the solution $\widetilde{\varphi}(y)$ of the initial value problem for (2.1) on $\left[-p_{0}, \delta\right]$ with the initial condition

$$
\widetilde{\varphi}\left(-p_{0}\right)=p_{0} \tan \beta_{1}+\sigma, \widetilde{\varphi}^{\prime}\left(-p_{0}\right)=\tan \left(\alpha_{1}+\varepsilon\right)
$$

for some positive constants $\delta, \varepsilon, \sigma$ with $\delta \in\left(0, R\left(p_{0}\right)\right)$ fixed. By the theory of continuous dependence, such a solution exists for $\varepsilon$ and $\sigma$ small. Also, $\widetilde{\varphi}$ can be extended to the left beyond $y=-p_{0}$ such that

$$
\widetilde{\varphi}(y)>(-y) \tan \beta_{1}, \widetilde{\varphi}^{\prime}(y)>\tan \alpha_{1}
$$

for all $y \in\left[-p_{0}-\gamma,-p_{0}\right]$ for some $\gamma>0$. Now, given any fixed $p \in\left(p_{0}, p_{0}+\gamma\right)$, it holds that $R(p) \leq 0$. Since

$$
\varphi(-p ; p)=p \tan \beta_{1}<\widetilde{\varphi}(-p), \varphi^{\prime}(-p ; p)=\tan \alpha_{1}<\widetilde{\varphi}^{\prime}(-p),
$$

it follows from the standard nonlinear comparison theorem [22, Theorem 23 of Chap. 1] that

$$
\varphi(y ; p) \leq \widetilde{\varphi}(y), \varphi^{\prime}(y ; p) \leq \widetilde{\varphi}^{\prime}(y)
$$

for all $y \in[-p, R)$, where $R:=\min \{R(p), \delta\}$. Then $R(p) \geq \delta>0$, a contradiction. Therefore, (2.16) is proved. 
Remark 2.1. By (2.13), we have

$$
\varphi(y ; p)>(y+p)\left(\tan \alpha_{1}+\tan \beta_{1}\right)-y \tan \beta_{1}>-y \tan \beta_{1}
$$

for $y \in(-p, R(p)) \cap(-p, 0]$. This shows that the graph of $\varphi$ is always above the left supporting line.

Since $\varphi^{\prime \prime}>0$, the limit

$$
l(p):=\lim _{y \rightarrow R(p)^{-}} \varphi^{\prime}(y ; p)
$$

exists and $l(p)>\tan \alpha_{1}$.

Lemma 2.4. Let $p \in\left(0, p_{0}\right)$. Then $R(p)=\infty$ and $l(p)<\infty$.

Proof. Recall that $R(p)>0$ for $p \in\left(0, p_{0}\right)$. Also,

$$
\lim _{q \rightarrow 0^{+}} \mathcal{H}(q, p)=\lim _{q \rightarrow 0^{+}} \frac{\varphi(q ; p)}{q}=\infty,
$$

since $\varphi(0 ; p)$ is finite for $p \in\left(0, p_{0}\right)$. If $R(p)<\infty$, then $\varphi(y ; p) \rightarrow \infty$ as $y \rightarrow R(p)^{-}$ and so

$$
\lim _{q \rightarrow R(p)^{-}} \mathcal{H}(q, p)=\lim _{q \rightarrow R(p)^{-}} \frac{\varphi(q ; p)}{q}=\infty,
$$

which contradicts the fact that $\mathcal{H}(q, p)$ is strictly decreasing in $q$. Hence $R(p)=\infty$.

Suppose that $l(p)=\infty$. Then $\varphi(y ; p) \rightarrow \infty$ as $y \rightarrow \infty$. By l'Hôpital's rule, we obtain that

$$
\lim _{q \rightarrow \infty} \mathcal{H}(q, p)=\lim _{q \rightarrow \infty} \frac{\varphi(q ; p)}{q}=\lim _{q \rightarrow \infty} \varphi^{\prime}(q ; p)=\infty,
$$

a contradiction. Hence $l(p)<\infty$ and the lemma is proved.

Lemma 2.5. Suppose $C \in\left(\tan \alpha_{1}, \infty\right)$. Then there exists a $p \in\left(0, p_{0}\right)$ such that

$$
\varphi^{\prime}(y ; p)<C, \forall y \in[-p, \infty) .
$$

Proof. Note that $\varphi(0 ; p)$ and $\varphi^{\prime}(0 ; p)$ are well defined for $p \in\left(0, p_{0}\right)$. Following from the continuity of $\varphi(0 ; p)$ and $\varphi^{\prime}(0 ; p)$ in $p$, we have $\varphi(0 ; p) \rightarrow 0$ and $\varphi^{\prime}(0 ; p) \rightarrow \tan \alpha_{1}$ as $p \downarrow 0^{+}$. Now, we choose $p \in\left(0, p_{0}\right)$ small enough such that

$$
\tan ^{-1} \varphi^{\prime}(0 ; p)+\varphi(0 ; p) \sqrt{\pi / 2}<\tan ^{-1} C .
$$

Then $\varphi^{\prime}(y ; p)<C$ for all $y \geq-p$. Indeed, from (2.5) it follows that

$$
(\rho(y) \mathcal{F}(y))^{\prime}=0, \quad \rho(y):=\exp \left\{\int_{0}^{y} s\left[1+\varphi^{\prime}(s)^{2}\right] d s\right\} .
$$

Hence we deduce that

$$
\left(\tan ^{-1}\left(\varphi^{\prime}(y ; p)\right)\right)^{\prime}=\mathcal{F}(y) \leq \mathcal{F}(0) e^{-y^{2} / 2}=\varphi(0 ; p) e^{-y^{2} / 2}, \quad y \in[0, \infty),
$$

and so

$$
\begin{aligned}
\tan ^{-1}\left(\varphi^{\prime}(y ; p)\right) & \leq \tan ^{-1}\left(\varphi^{\prime}(0 ; p)\right)+\varphi(0 ; p) \int_{0}^{y} e^{-t^{2} / 2} d t \\
& \leq \tan ^{-1}\left(\varphi^{\prime}(0 ; p)\right)+\varphi(0 ; p) \sqrt{\pi / 2} \\
& <\tan ^{-1} C
\end{aligned}
$$

for all $y \in[0, \infty)$. Then the lemma is proved. 
Now, we set $p_{*}:=\sup A$ and $p^{*}:=\sup B$, where

$$
\begin{aligned}
& A:=\left\{p \in\left(0, p_{0}\right) \mid \varphi^{\prime}(y ; p)<\tan \alpha_{2}, \forall y \in[-p, \infty)\right\}, \\
& B:=\left\{p \in\left(0, p_{0}\right) \mid \varphi^{\prime}(0 ; p)<\tan \alpha_{2}\right\}
\end{aligned}
$$

for a given $\alpha_{2} \in\left(\alpha_{1}, \pi / 2\right)$. Notice that $A \subset B$ and, by Lemma $2.5 A$ is nonempty. Hence $p_{*}$ and $p^{*}$ are well defined and $0<p_{*} \leq p^{*} \leq p_{0}$.

We first claim that $p^{*}<p_{0}$. For contradiction, we assume that $p^{*}=p_{0}$. Let $I:=\left[-p_{0}, 0\right]$. Then for any $y \in I$ we have

$$
\varphi^{\prime}(y ; p) \leq \varphi^{\prime}(0 ; p)<\tan \alpha_{2}, \forall p \in\left(0, p_{0}\right) .
$$

By letting $p \uparrow p_{0}$, we obtain that

$$
\varphi^{\prime}\left(y ; p_{0}\right) \leq \tan \alpha_{2}, \forall y \in I .
$$

Therefore, $\varphi\left(y ; p_{0}\right)$ can be extended over $y=0$ and so $R\left(p_{0}\right)>0$. This contradicts (2.16). Hence $p^{*}<p_{0}$.

Next, we claim that

$$
\varphi^{\prime}\left(0 ; p^{*}\right)=\tan \alpha_{2} .
$$

Recall that $R(p)>0$ for $p \in\left(0, p_{0}\right)$. It follows from the definition of $p^{*}$ and the continuity of $\varphi^{\prime}(0 ; p)$ at $p=p^{*}$ that $\varphi^{\prime}\left(0 ; p^{*}\right) \leq \tan \alpha_{2}$. Suppose that $\varphi^{\prime}\left(0 ; p^{*}\right)<$ $\tan \alpha_{2}$. Then again from the continuity of $\varphi^{\prime}(0 ; p)$ it follows that $\varphi^{\prime}(0 ; p)<\tan \alpha_{2}$ for $p \in\left(p^{*}-\delta, p^{*}+\delta\right)$ for some $\delta>0$. This is a contradiction. Hence (2.17) follows.

Now, we choose $p_{n} \in A$ such that $p_{n} \uparrow p_{*}$ as $n \rightarrow \infty$. From $\varphi^{\prime}\left(1 ; p_{n}\right)<\tan \alpha_{2}$ for all $n$ it follows that $\varphi^{\prime}\left(1 ; p_{*}\right) \leq \tan \alpha_{2}$. Hence $\varphi^{\prime}\left(0 ; p_{*}\right)<\tan \alpha_{2}$ and $p_{*} \in B$. Therefore, $p_{*}<p^{*}$, since $B$ is an open set.

Notice that the constants $p_{0}, p^{*}, p_{*}$ depend only on $\beta_{1}, \alpha_{1}, \alpha_{2}$.

Lemma 2.6. Given $\beta_{1} \in[0, \pi / 2), \alpha_{1} \in\left(-\beta_{1}, \pi / 2\right)$, and $\alpha_{2}>\alpha_{1}$. Then for each $p \in\left(p_{*}, p^{*}\right)$ there exists a unique $q=q(p)>0$ such that $\mathcal{G}(q, p)=\tan \alpha_{2}$. Moreover, the function $q(p)$ is a strictly decreasing continuous function of $p$ in $\left(p_{*}, p^{*}\right)$ such that $q(p) \rightarrow \infty$ as $p \rightarrow p_{*}^{+}$and $q(p) \rightarrow 0$ as $p \rightarrow\left(p^{*}\right)^{-}$.

Proof. For any $p \in\left(p_{*}, p^{*}\right)$, we have $\mathcal{G}(0, p)<\tan \alpha_{2}$ and $\mathcal{G}(q ; p) \geq \tan \alpha_{2}$ for some $q>0$. Hence the function $q(p)$ is well defined on $\left(p_{*}, p^{*}\right)$. By the theory of continuous dependence, $q$ is continuous. The monotonicity of $q$ follows from (2.11) and (2.15). By (2.17), $q(p) \rightarrow 0$ as $p \rightarrow\left(p^{*}\right)^{-}$. Suppose that $K:=\lim _{p \rightarrow p_{*}^{+}} q(p)<\infty$. Then $\varphi^{\prime}(K ; p) \geq \varphi^{\prime}(q(p) ; p)=\tan \alpha_{2}$ for any $p \in\left(p_{*}, p^{*}\right)$. Hence $\varphi^{\prime}\left(K ; p_{*}\right) \geq \tan \alpha_{2}$ and so $\varphi^{\prime}\left(K+1 ; p_{*}\right)>\tan \alpha_{2}$. But, by the definition of $p_{*}$, we have $\varphi^{\prime}\left(y ; p_{*}\right) \leq$ $\tan \alpha_{2}$ for any $y \in\left[-p_{*}, \infty\right)$, a contradiction. Hence the lemma is proved.

Now, we define $\widehat{p}:=\sup \widehat{A}$, where

$$
\widehat{A}:=\left\{p \in\left(0, p_{0}\right) \mid \mathcal{H}(q, p)=\tan \beta_{2} \text { for some } q \in(0, \infty)\right\} .
$$

First, we show that the set $\widehat{A}$ is open. For this purpose, let $\widetilde{p} \in \widehat{A}$. Then there is $q \in(0, \infty)$ such that $\mathcal{H}(q, \widetilde{p})=\tan \beta_{2}$. Recall that $\mathcal{H}(q, \widetilde{p})=\varphi(q ; \widetilde{p}) / q$. It follows from the strictly decreasing property of $\mathcal{H}$ in $q$ that $\mathcal{H}(q+1, \widetilde{p})<\tan \beta_{2}$, i.e., $\varphi(q+1 ; \widetilde{p})<(q+1) \tan \beta_{2}$. Then by the theory of continuous dependence there is a positive constant $\delta$ such that $\varphi(q+1 ; p)<(q+1) \tan \beta_{2}$ for all $p \in(\widetilde{p}-\delta, \widetilde{p}+\delta)$. On the other hand, $\lim _{q \rightarrow 0^{+}} \mathcal{H}(q, p)=\infty$ for $p \in\left(0, p_{0}\right)$, since $\varphi(0 ; p)$ is finite for $p \in\left(0, p_{0}\right)$. This implies that for each $p \in(\widetilde{p}-\delta, \widetilde{p}+\delta)$ there is a $Q=Q(p) \in(0, q+1)$ such that $\mathcal{H}(Q, p)=\tan \beta_{2}$. Hence $\widehat{A}$ is open. 
Next, for $p=p_{*}$ and $q>0$

$$
\varphi(q ; p)=p \tan \beta_{1}+\int_{-p}^{q} \varphi^{\prime}(y ; p) d y \leq p \tan \beta_{1}+\left(\tan \alpha_{2}\right)(q+p),
$$

we get

$$
\lim _{q \rightarrow \infty} \mathcal{H}\left(q, p_{*}\right)=\lim _{q \rightarrow \infty} \frac{\varphi\left(q ; p_{*}\right)}{q} \leq \tan \alpha_{2}<\tan \beta_{2} .
$$

Also, $\lim _{q \rightarrow 0^{+}} \mathcal{H}\left(q, p_{*}\right)=\infty$. It follows that there is a $q_{*}:=Q\left(p_{*}\right)>0$ such that $\mathcal{H}\left(q_{*}, p_{*}\right)=\tan \beta_{2}$. Hence $p_{*} \in \widehat{A}$ and $p_{*}<\widehat{p}$.

Note that $\widehat{p}$ depends on $\alpha_{1}, \alpha_{2}, \beta_{1}, \beta_{2}$.

Lemma 2.7. Given $\beta_{1}, \beta_{2} \in[0, \pi / 2), \alpha_{1} \in\left(-\beta_{1}, \pi / 2\right)$, and $\alpha_{2} \in\left(\alpha_{1}, \beta_{2}\right)$. Then for each $p \in\left(p_{*}, \widehat{p}\right)$ there is a unique $Q=Q(p)>0$ such that $\mathcal{H}(Q(p), p)=\tan \beta_{2}$. Furthermore, $Q(p)$ is strictly increasing in $p$ with $Q\left(p_{*}\right)<\infty$ and $Q(p) \rightarrow \infty$ as $p \rightarrow \widehat{p}^{-}$.

Proof. From (2.11), (2.15) and the definition of $\widehat{p}$ it follows that for each $p \in\left(p_{*}, \widehat{p}\right)$ there is a unique $Q=Q(p)>0$ such that $\mathcal{H}(Q(p), p)=\tan \beta_{2}$. The strictly increasing property of $Q$ follows from (2.11) and (2.15).

Let $L:=\lim _{p \rightarrow \widehat{p}^{-}} Q(p)$. If $L<\infty$, then by the continuity of $\mathcal{H}$ we have $\mathcal{H}(L, \widehat{p})=$ $\tan \beta_{2}$. This implies that $\widehat{p} \in \widehat{A}$, contradicting the definition of $\widehat{p}$ and the fact that $\widehat{A}$ is open. The proof is completed.

We are ready to prove the main theorem of this section as follows.

Theorem 2.8. Given $\alpha_{1}, \alpha_{2}, \beta_{1}, \beta_{2}$ with $\beta_{1}, \beta_{2} \in[0, \pi / 2), \alpha_{1} \in\left(-\beta_{1}, \pi / 2\right)$, and $\alpha_{2} \in\left(\alpha_{1}, \beta_{2}\right)$. Then there is a unique solution $(\varphi(y), p, q)(p, q>0)$ of the two-point free boundary problem (2.1), (2.2) and (2.3).

Proof. It follows from Lemmas 2.6 and 2.7 that there is a unique positive pair $(\bar{p}, \bar{q})$ such that $\bar{q}=q(\bar{p})=Q(\bar{p})$. Then the triple $(\varphi(y ; \bar{p}), \bar{p}, \bar{q})$ is the desired unique solution of the two-point free boundary problem (2.1), (2.2) and (2.3).

\section{Asymptotic stability}

In this section, we shall study the asymptotic stability of the self-similar expanding curve obtained in $\S 2$. Recall

$$
S(t):=\{(z, U(z, t)) \mid-\sqrt{2(t+1)} p \leq z \leq \sqrt{2(t+1)} q\} .
$$

We obtain the following stability theorem.

Theorem 3.1. Assume that $\alpha_{1}<\alpha_{2}, u_{0} \in C^{2}\left[-\xi_{01}, \xi_{02}\right]$ satisfying the compatibility conditions, and $u_{0}>0$ in $\left(-\xi_{01}, \xi_{02}\right)$. Let $\Gamma(t)$ be the curve corresponding to a solution of (1.2) with the initial curve $\Gamma_{0}=\left\{\left(x, u_{0}(x)\right) \mid-\xi_{01} \leq x \leq \xi_{02}\right\}$ and $S(t)$ be the self-similar expanding curve obtained in $\S 2$. Then $S(t)$ is asymptotically stable in the sense:

$$
d_{H}(\Gamma(t), S(t)) \leq C t^{-\delta_{*}}, \quad t \geq 1,
$$

for some $\delta_{*}>0$ and $C$ a positive constant independent of $t$.

Here $d_{H}$ (which is equivalent to the Hausdorff distance) is defined by

$$
d_{H}(\Gamma(t), S(t)):=\sup _{x \in E_{1}(z, t), z \in E_{2}(t)}\left|\sqrt{x^{2}+[u(x, t)]^{2}}-\sqrt{z^{2}+[U(z, t)]^{2}}\right|,
$$


where

$$
\begin{aligned}
& E_{1}(z, t):=\left\{x \mid x U(z, t)=z u(x, t),-\xi_{1}(t) \leq x \leq \xi_{2}(t)\right\}, \\
& E_{2}(t):=\{z \mid-\sqrt{2(t+1)} p \leq z \leq \sqrt{2(t+1)} q\} .
\end{aligned}
$$

This theorem and its proof are the same as the one given in [12] for the heat equation case. We only outline the idea and omit the detail of its proof here.

First, we introduce the following similarity change of variables:

$$
\begin{aligned}
& w(\eta, \tau)=\frac{u(x, t)}{\sqrt{2(t+1)}}, \eta=\frac{x}{\sqrt{2(t+1)}}, \tau=\ln \sqrt{2(t+1)}, \\
& \psi_{1}(\tau)=\frac{\xi_{1}(t)}{\sqrt{2(t+1)}}, \psi_{2}(\tau)=\frac{\xi_{2}(t)}{\sqrt{2(t+1)}} .
\end{aligned}
$$

By this change of variables, the problem (1.2) is transformed into

$$
\left\{\begin{array}{c}
w_{\tau}=w_{\eta \eta} /\left(1+w_{\eta}^{2}\right)+\eta w_{\eta}-w,-\psi_{1}(\tau)<\eta<\psi_{2}(\tau), \tau>\ln \sqrt{2}=: \tau_{0} \\
w_{\eta}\left(-\psi_{1}(\tau), \tau\right)=\tan \alpha_{1}, w\left(-\psi_{1}(\tau), \tau\right)=\psi_{1}(\tau) \tan \beta_{1}, \tau>\tau_{0} \\
w_{\eta}\left(\psi_{2}(\tau), \tau\right)=\tan \alpha_{2}, w\left(\psi_{2}(\tau), \tau\right)=\psi_{2}(\tau) \tan \beta_{2}, \tau>\tau_{0}
\end{array}\right.
$$

with the initial condition

$$
\left\{\begin{array}{l}
w\left(\eta, \tau_{0}\right)=u_{0}(\sqrt{2} \eta) / \sqrt{2}=: w_{0}(\eta),-\psi_{01} \leq \eta \leq \psi_{02} \\
\psi_{1}\left(\tau_{0}\right)=\xi_{01} / \sqrt{2}=: \psi_{01}, \psi_{2}\left(\tau_{0}\right)=\xi_{02} / \sqrt{2}=: \psi_{02}
\end{array}\right.
$$

Using the self-similar solution $\varphi$ obtained in $\S 2$, we construct a pair of sub-supersolutions such that they converge to the solution $\varphi$. Finally, by returning to the original variables, we deduce Theorem 3.1.

\section{ACKNOWLEDGMENT}

This work was partially supported by National Science Council of the Republic of China under the contracts NSC-90-2115-M-133-001, NSC 90-2115-M-003-009, and NSC 90-2115-M-122-003. The authors thank the referee for many helpful comments.

\section{REFERENCES}

[1] S. J. Altschuler and L. F. Wu, Convergence to translating solutions for a class of quasilinear parabolic boundary problems, Math. Ann. 295 (1993), 761-765. MR 94d:35082

[2] S. J. Altschuler and L. F. Wu, Translating surfaces of the non-parametric mean curvature flow with prescribed contact angle, Calc. Var. 2 (1994), 101-111. MR 97b:58032

[3] J. D. Buckmaster and G. S. S. Ludford, Theory of Laminar Flames, Cambridge University Press, Cambride, 1982. MR 84f:80011

[4] W. K. Burton, N. Cabrera, and F. C. Frank, The growth of crystals and equilibrium structure of their surfaces, Philos. Trans. Roy. Soc. London A 243 (1951), 299-358. MR 13:196f

[5] L. A. Caffarelli and J. L. Vazquez, A free boundary problem for the heat equation arising in flame propagation, Trans. Amer. Math. Soc. 347 (1995), 411-441. MR 95e:35097

[6] Y.-G. Chen, Y. Giga, and S. Goto, Uniqueness and existence of viscosity solutions of generalized mean curvature flow equations, J. Diff. Geometry 33 (1991), 749-786. MR 93a:35093

[7] B. Chow and D. H. Tsai, Geometric expansion of convex plane curves, J. Diff. Geom. 44 (1996), 312-330. MR 97m:58041

[8] K. Deckelnick, C. M. Elliott, and G. Richardson, Long time asymptotics for forced curvature flow with applications to the motion of a superconducting vortex, Nonlinearity 10 (1997), 665-678. MR 98b:35080

[9] L. C. Evans and J. Spruck, Motion of level sets by mean curvature 1, J. Diff. Geom. 33 (1991), 635-681. MR 92h:35097 
[10] V. A. Galaktionov, J. Hulshof and J. L. Vazquez, Extinction and focusing behaviour of spherical and annular flames described by a free boundary problem, J. Math. Pures Appl. 76 (1997), 563-608. MR 98h:35238

[11] Y. Giga, N. Ishimura, and Y. Kohsaka, Spiral solutions for a weakly anisotropic curvature flow equation, Hokkaido University Preprint Series in Mathematics, Series \#529, June 2001.

[12] J.-S. Guo and Y. Kohsaka, Two-point free boundary problem for heat equation, preprint.

[13] M. Gurtin, Thermomechanics of Evolving Phase Boundaries in the Plane, Clarendon Press, Oxford, UK, 1993. MR 97k:73001

[14] D. Hilhorst and J. Hulshof, A free boundary focusing problem, Proc. Amer. Math. Soc. 121 (1994), 1193-1202. MR 94j:35200

[15] G. Huisken, Non-parametric mean curvature evolution with boundary conditions, J. Diff. Equations 77 (1989), 369-378. MR 90g:35050

[16] H. Imai, N. Ishimura, and T. Ushijima, A crystalline motion of spiral-shaped curves with symmetry, J. Math. Anal. Appl. 240 (1999), 115-127. MR 2000j:53091

[17] J. Keener and J. Sneyd, Mathematical Physiology, Springer-Verlag, New York, 1998. MR 2000c:92010

[18] Y. Kohsaka, Free boundary problem for quasilinear parabolic equation with fixed angle of contact to a boundary, Nonlinear Analysis 45 (2001), 865-894. MR 2002j:35320

[19] K. Mikula and D. Ševčovič, Evolution of plane curves driven by a nonlinear function of curvatures and anisotropy, SIAM J. Appl. Math. 61 (2001), 1473-1501. MR 2002b:65181

[20] H. Ninomiya and M. Taniguchi, Traveling curved fronts of a mean curvature flow with constant driving force, Free Boundary Problems: Theory and Applications I, Mathematical Sciences and Applications 13, Gakuto International Series, 2000, pp. 206-221. MR 2001j:53093

[21] H. Ninomiya and M. Taniguchi, Stability of traveling curved fronts in a curvature flow with driving force, Methods and Applications of Analysis 8 (2001), 429-450. MR 2003c:35012]

[22] M. H. Protter and H. F. Weinberger, Maximum Principles in Differential Equations, Springer-Verlag, 1984. MR 86f:35034

[23] J. A. Sethian, Level Set Methods and Fast Marching Methods: Evolving Interfaces in Computational Geometry, Fluid Mechanics, Computer Vision, and Materials Science, Cambridge University Press, Cambridge, UK, 1999. MR 2000c:65015

[24] J. L. Vazquez, The free boundary problem for the heat equation with fixed gradient condition, Free boundary problems, theory and applications, Zakopane, Poland, Pitman Res. Notes in Math. Series 363, 1995, pp. 277-302. MR 98h:35246

Department of Computer and Information Sciences, National Taiwan Ocean UniverSity, 2, Pei-Ning Road, Keelung, Taiwan

E-mail address: felix@cs.ntou.edu.tw

Department of Mathematics, National Taiman Normal University, 88, S-4 Ting Chou ROAD, TAIPEI 117 , TAIWAN

E-mail address: jsguo@math.ntnu.edu.tw

Department of Applied Mathematics, Providence University, 200, Chung-Chi Road, Shalu, Taichung County 433, Taiwan

E-mail address: cplo@pu.edu.tw 